\title{
Magnetic Symmetry of the Plain Domain Walls in the Plates of Cubic Ferro- and Ferrimagnets
}

\author{
B.M. TANYGIN ${ }^{a, *}$ AND O.V. TYCHKO ${ }^{b}$ \\ Kyiv Taras Shevchenko National University, Radiophysics Faculty \\ Volodumiurska 64, Kyiv, MSP 01601, Ukraine \\ Magnetic symmetry of possible plane domain walls in arbitrary oriented plates of the crystal of hexoctahedral \\ crystallographic class is considered. The symmetry classification is applied for ferro- and ferrimagnets.
}

PACS numbers: 61.50.Ah, 75.60.Ch

\section{Introduction}

For sequential examination of static and dynamic properties of domain walls (DWs) in magnetically ordered media it is necessary to take into account their magnetic symmetry $[1,2]$. The complete symmetry classification of plane $180^{\circ}$-DWs in magnetically ordered crystals [1], similar classification of these DWs with the Bloch lines in ferromagnets and ferrites [2] and magnetic symmetry classification of plane non- $180^{\circ}$-DWs (all possible DW types including $0^{\circ}$-DWs [3]) in ferro- and ferrimagnets [4] were carried out earlier. These DW symmetry classifications allows arbitrary crystallographic point symmetry group of the crystal. The influence of the spatially restricted sample surfaces on the DW magnetic symmetry was not considered in works [1-4]. The real magnetic sample restricts a spatial (3D) magnetization distribution. Therefore, it modifies the DW symmetry in general case. This paper presents the investigation of the influence of the restricted sample surfaces on the symmetry of the all possible $\left(0^{\circ}-, 60^{\circ}-, 70.5^{\circ}-, 90^{\circ}-, 109.5^{\circ}-, 120^{\circ}-\right.$ and $180^{\circ}$-DW $[4,5]$ ) plane (i.e., DW with $r_{0} \gg \delta$, where $r_{0}$ is the curvature radius of the DW [1]) DWs in an arbitrary oriented plate of the cubic (crystallographic point symmetry group $m \overline{3} m$ ) ferro- and ferrimagnets.

\section{Domain wall symmetry in the restricted sample}

The DW symmetry can be described by the magnetic symmetry classes (MSCs) $G_{k}$ where $k$ is a MSC number [1]. The MSC $G_{k}$ of DW is the magnetic symmetry group including all symmetry transformations (all translations are considered as unit operations) that do not change the spatial distribution of magnetic moments in

* corresponding author; e-mail: b.m.tanygin@gmail.com the crystal with DW. The above-mentioned group is a subgroup of the magnetic (Shubnikov) symmetry group $G_{P}^{\infty}$ of the crystal paramagnetic phase [6]. Total number of MSCs of arbitrary type DWs (i.e., DWs with arbitrary $2 \alpha$ angle $\left(0^{\circ} \leq 2 \alpha \leq 180^{\circ}\right)$ between the unit time-odd axial vectors $\boldsymbol{m}_{1}$ and $\boldsymbol{m}_{2}$ directed along magnetization vectors $M_{1}$ and $M_{2}$ in neighbouring domains) in ferroand ferrimagnets is equal to 64 . General enumeration of MSCs contains $42 \mathrm{MSCs}(1 \leq k \leq 42)$ of $180^{\circ}$-DWs [1], 10 MSCs $(7 \leq k \leq 13$ and $16 \leq k \leq 18)$ of non $0^{\circ} / 180^{\circ}$-DWs and 42 MSCs $(k=2,6 \leq k \leq 13,16 \leq k \leq 19, k=22$, $24,26,30,32,37,39$ and $43 \leq k \leq 64)$ of $0^{\circ}$-DWs [4]. The MSCs $(k=25,28,37-41,52,54,61-63)$ with sixfold symmetry axes (including inversion axes) do not realize in the cubic crystals [4].

The unified co-ordinate system $O x y z$ is chosen as $\left[\boldsymbol{e}_{\tilde{x}}, \boldsymbol{e}_{\tilde{y}}, \boldsymbol{e}_{\tilde{z}}\right]=\left[\boldsymbol{a}_{2},-\boldsymbol{a}_{1}, \boldsymbol{n}_{\mathrm{W}}\right]$ where $\boldsymbol{n}_{\mathrm{W}}$ is the unit polar time-even vector along the DW plane normal [4]. For the $180^{\circ}$-DWs the vectors $\boldsymbol{a}_{1}$ and $\boldsymbol{a}_{2}$ are given earlier [1] as vectors $\boldsymbol{\tau}_{1}$ and $\boldsymbol{\tau}_{2}$, respectively. For the case of $2 \alpha \neq 180^{\circ}$ the unit vector $\boldsymbol{a}_{1}$ coincides with the direction of the vector $\Delta \boldsymbol{m}-\boldsymbol{n}_{\mathrm{W}}\left(\boldsymbol{n}_{\mathrm{W}} \Delta \boldsymbol{m}\right)$ (at $b_{\Delta} \neq 0$ and $\left.b_{\Sigma}=0\right)$ or $\left[\boldsymbol{a}_{2} \times \boldsymbol{n}_{\mathrm{W}}\right]\left(\right.$ at $b_{\Delta}=0$ or $\left.b_{\Sigma} \neq 0\right)$ where $\Delta \boldsymbol{m}=\boldsymbol{m}_{2}-\boldsymbol{m}_{1}, b_{\Delta}=\left|\left[\boldsymbol{n}_{\mathrm{W}} \times \Delta \boldsymbol{m}\right]\right|, b_{\Sigma}=\left|\left[\boldsymbol{n}_{\mathrm{W}} \times \boldsymbol{m}_{\Sigma}\right]\right|$. Here the unit vector $\boldsymbol{a}_{2}$ coincides with the direction of the vector $\boldsymbol{m}_{\Sigma}-\boldsymbol{n}_{\mathrm{W}}\left(\boldsymbol{n}_{\mathrm{W}} \boldsymbol{m}_{\Sigma}\right)\left(\right.$ at $\left.b_{\Sigma} \neq 0\right)$ or $\left[\boldsymbol{n}_{\mathrm{W}} \times \boldsymbol{a}_{1}\right]$ (at $b_{\Delta} \neq 0$ and $b_{\Sigma}=0$ ), or else, with an arbitrary direction in the DW plane $\left(\boldsymbol{a}_{2} \perp \boldsymbol{n}_{\mathrm{W}}\right.$ at $\left.b_{\Sigma}=b_{\Delta}=0\right)$ where $\boldsymbol{m}_{\Sigma}=\boldsymbol{m}_{1}+\boldsymbol{m}_{2}$. The mutual orientation of the vectors $\boldsymbol{m}_{1}, \boldsymbol{m}_{2}$ and $\boldsymbol{n}_{\mathrm{W}}$ is determined by the parameters: $a_{\Sigma}=\left(\boldsymbol{n}_{\mathrm{W}} \boldsymbol{m}_{\Sigma}\right), a_{\Delta}=\left(\boldsymbol{n}_{\mathrm{W}} \Delta \boldsymbol{m}\right), a_{\mathrm{C}}=\left(\boldsymbol{n}_{\mathrm{W}} \boldsymbol{m}_{\mathrm{C}}\right)$, $b_{\Sigma}$ and $b_{\Delta}$, where $\boldsymbol{m}_{\mathrm{C}}=\left[\boldsymbol{m}_{1} \times \boldsymbol{m}_{2}\right]$. The mutual orientation of the vectors $\boldsymbol{m}_{1}, \boldsymbol{m}_{2}, \boldsymbol{n}_{\mathrm{W}}$ and $\boldsymbol{n}_{S}$ is determined by parameters: $a_{1}=\left(\boldsymbol{a}_{1} \boldsymbol{n}_{S}\right), a_{2}=\left(\boldsymbol{a}_{2} \boldsymbol{n}_{S}\right)$, $a_{n}=\left(\boldsymbol{n}_{\mathrm{W}} \boldsymbol{n}_{S}\right), b_{1}=\left|\left[\boldsymbol{n}_{S} \times \boldsymbol{a}_{1}\right]\right|, b_{2}=\left|\left[\boldsymbol{n}_{S} \times \boldsymbol{a}_{2}\right]\right|$ and $b_{n}=\left|\left[\boldsymbol{n}_{S} \times \boldsymbol{n}_{\mathrm{W}}\right]\right|$, where $\boldsymbol{n}_{S}$ is sample plane normal.

The MSC $G_{P}$ of restricted sample of crystal in paramagnetic phase could be defined as $G_{P}=G_{P}^{\infty} \cap G_{S}$ where 
the sample shape MSC $G_{S}$ is $\infty / m m m 1^{\prime}$ for volumetric plate. MSCs of DWs in volumetric plate should satisfy the condition $G_{k} \subset G_{P}$. The MSCs of the all possible plane DWs in the arbitrary oriented plate of cubic crystals of hexoctahedral class (crystallographic point symmetry group $m \overline{3} m$ in the paramagnetic phase [6]) are presented in Table. Here, symmetry axes are collinear with vectors $\boldsymbol{a}_{1}$ and $\boldsymbol{a}_{2}$ and reflection planes are perpendicular to them. For MSCs with: $k=24, k=26$, $k=27 ; 29 \leq k \leq 36 ; 42 \leq k \leq 51, k=53,55 \leq k \leq 60$ and $k=64$ only generative symmetry elements are represented.

TABLE

MSCs of the plane $2 \alpha$-DWs in plates of the cubic $m \overline{3} m$ crystal.

\begin{tabular}{|c|c|c|c|c|c|}
\hline$k$ & $\{n m l\}$-sample & $\begin{array}{l}\boldsymbol{m}_{1}, \boldsymbol{m}_{2}, \boldsymbol{n}_{\mathrm{W}} \text { and } \boldsymbol{n}_{S}: \\
\text { mutual orientation** }\end{array}$ & $\begin{array}{l}\boldsymbol{m}_{1}, \boldsymbol{m}_{2} \text { and } \boldsymbol{n}_{\mathrm{W}}: \\
\text { mutual orientation }\end{array}$ & $\begin{array}{c}\text { Symmetry } \\
\text { elements }\end{array}$ & $\begin{array}{c}\text { MSC } \\
\text { symbol }\end{array}$ \\
\hline 1 & $\{100\},\{110\}$ & $b_{n}=0$ or $b_{1}=0$ & $a_{\Sigma}=b_{\Sigma}=a_{\Delta}=0$ & $\left(1,2_{2}, \overline{2}_{1}, \overline{2}_{n}\right) \times(1, \overline{1})$ & $\mathrm{mmm}$ \\
\hline 2 & $\{100\},\{110\}$ & $b_{n}=0$ or $b_{1}=0$ & $\begin{array}{c}a_{\Delta}=b_{\Delta}=a_{\Sigma}=0 \\
\text { or } a_{\Sigma}=b_{\Sigma}=a_{\Delta}=0\end{array}$ & $1, \overline{2}_{1}^{\prime}, \overline{2}_{2}, 2_{n}^{\prime}$ & $m m^{\prime} 2^{\prime}$ \\
\hline 3 & $\{100\},\{110\}$ & $b_{n}=0$ or $b_{1}=0$ & $a_{\Sigma}=b_{\Sigma}=a_{\Delta}=0$ & $1,2_{2}, \overline{2}_{1}, \overline{2}_{n}$ & $m m 2$ \\
\hline 4 & $\{n m l\}^{*}$ & $a_{1}=0$ or $b_{1}=0$ & $a_{\Sigma}=b_{\Sigma}=a_{\Delta}=0$ & $1, \overline{1}^{\prime}, 2_{1}^{\prime}, \overline{2}_{1}$ & $2^{\prime} / m$ \\
\hline 5 & $\{n m l\}^{*}$ & $a_{n}=0$ or $b_{n}=0$ & $a_{\Sigma}=b_{\Sigma}=a_{\Delta}=0$ & $1, \overline{1}^{\prime}, 2_{n}^{\prime}, \overline{2}_{n}$ & $2^{\prime} / m$ \\
\hline 6 & $\{n m l\}^{*}$ & $a_{2}=0$ or $b_{2}=0$ & $a_{\Sigma}=a_{\Delta}=a_{\mathrm{C}}=0$ & $1, \overline{2}_{2}$ & $m$ \\
\hline 7 & $\{100\},\{110\}$ & $b_{n}=0$ or $b_{1}=0$ & $a_{\Sigma}=a_{\Delta}=0$ & $1,2_{1}^{\prime}, 2_{2}, 2_{n}^{\prime}$ & $22^{\prime} 2^{\prime}$ \\
\hline 8 & $\{n m l\}^{*}$ & $a_{n}=0$ or $b_{n}=0$ & $a_{\Sigma}=a_{\Delta}=0$ & $1,2_{n}^{\prime}$ & $2^{\prime}$ \\
\hline 9 & $\{100\},\{110\}$ & $b_{n}=0$ or $b_{1}=0$ & $a_{\mathrm{C}}=a_{\Delta}=b_{\Sigma}=0$ & $1,2_{1}^{\prime}, \overline{2}_{2}^{\prime}, \overline{2}_{n}$ & $m m^{\prime} 2^{\prime}$ \\
\hline 10 & $\{n m l\}^{*}$ & $a_{1}=0$ or $b_{1}=0$ & $a_{\Delta}=0$ & $1,2_{1}^{\prime}$ & $2^{\prime}$ \\
\hline 11 & $\{n m l\}^{*}$ & $a_{n}=0$ or $b_{n}=0$ & $a_{\mathrm{C}}=a_{\Delta}=b_{\Sigma}=0$ & $1, \overline{2}_{n}$ & $m$ \\
\hline 12 & $\{n m l\}^{*}$ & $a_{1}=0$ or $b_{1}=0$ & $a_{\mathrm{C}}=0$ & $1, \overline{2}_{1}^{\prime}$ & $m^{\prime}$ \\
\hline 13 & $\{n m l\}^{*}$ & $a_{2}=0$ or $b_{2}=0$ & $a_{\Sigma}=0$ & $1,2_{2}$ & 2 \\
\hline 14 & $\{n m l\}^{*}$ & $a_{2}=0$ or $b_{2}=0$ & $a_{\Sigma}=b_{\Sigma}=0$ & $1, \overline{1}, 2_{2}^{\prime}, \overline{2}_{2}^{\prime}$ & $2^{\prime} / m^{\prime}$ \\
\hline 15 & arbitrary & arbitrary & $a_{\Sigma}=b_{\Sigma}=0$ & $1, \overline{1}^{\prime}$ & $\overline{1}^{\prime}$ \\
\hline 16 & arbitrary & arbitrary & arbitrary & 1 & 1 \\
\hline 17 & $\{100\},\{110\}$ & $b_{n}=0$ or $b_{1}=0$ & $a_{\mathrm{C}}=a_{\Sigma}=b_{\Delta}=0$ & $1, \overline{2}_{1}^{\prime}, 2_{2}, \overline{2}_{n}^{\prime}$ & $m^{\prime} m^{\prime} 2$ \\
\hline 18 & $\{n m l\}^{*}$ & $a_{n}=0$ or $b_{n}=0$ & $a_{\mathrm{C}}=a_{\Sigma}=b_{\Delta}=0$ & $1, \overline{2}_{n}^{\prime}$ & $m^{\prime}$ \\
\hline 19 & $\{n m l\}^{*}$ & $a_{n}=0$ or $b_{n}=0$ & $b_{\Delta}=b_{\Sigma}=0$ & $1,2_{n}$ & 2 \\
\hline 20 & $\{n m l\}^{*}$ & $a_{n}=0$ or $b_{n}=0$ & $a_{\Sigma}=b_{\Sigma}=b_{\Delta}=0$ & $1, \overline{1}^{\prime}, 2_{n}, \overline{2}_{n}^{\prime}$ & $2 / m$ \\
\hline 21 & $\{100\},\{110\}$ & $b_{n}=0$ or $b_{1}=0$ & $a_{\Sigma}=b_{\Sigma}=b_{\Delta}=0$ & $1,2_{1}, 2_{2}, 2_{n}$ & 222 \\
\hline 22 & $\{100\},\{110\}$ & $b_{n}=0$ or $b_{1}=0$ & $b_{\Delta}=b_{\Sigma}=0$ & $1, \overline{2}_{1}^{\prime}, \overline{2}_{2}^{\prime}, 2_{n}$ & $m^{\prime} m^{\prime} 2$ \\
\hline 23 & $\{100\},\{110\}$ & $b_{n}=0$ or $b_{1}=0$ & $a_{\Sigma}=b_{\Sigma}=b_{\Delta}=0$ & $\left(1,2_{1}, 2_{2}, 2_{n}\right) \times\left(1, \overline{1}^{\prime}\right)$ & $m^{\prime} m^{\prime} m^{\prime}$ \\
\hline 24 & $\{111\}$ & $b_{n}=0$ & $b_{\Delta}=b_{\Sigma}=0$ & $3_{n}$ & 3 \\
\hline 26 & $\{111\}$ & $b_{n}=0$ & $b_{\Delta}=b_{\Sigma}=0$ & $3_{n}, \overline{2}_{1}^{\prime}$ & $3 m^{\prime}$ \\
\hline 27 & $\{111\}$ & $b_{n}=0$ & $a_{\Sigma}=b_{\Sigma}=b_{\Delta}=0$ & $3_{n}, 2_{1}$ & 32 \\
\hline 29 & $\{111\}$ & $b_{n}=0$ & $a_{\Sigma}=b_{\Sigma}=b_{\Delta}=0$ & $\overline{3}_{n}^{\prime}, \overline{2}_{1}^{\prime}$ & $\overline{3}^{\prime} m^{\prime}$ \\
\hline 30 & $\{100\}$ & $b_{n}=0$ & $b_{\Delta}=b_{\Sigma}=0$ & $4_{n}$ & 4 \\
\hline 31 & $\{100\}$ & $b_{n}=0$ & $a_{\Sigma}=b_{\Sigma}=b_{\Delta}=0$ & $4_{n}, \overline{2}_{n}^{\prime}$ & $4 / m^{\prime}$ \\
\hline 32 & $\{100\}$ & $b_{n}=0$ & $b_{\Delta}=b_{\Sigma}=0$ & $4_{n}, \overline{2}_{1}^{\prime}$ & $4 m^{\prime} m^{\prime}$ \\
\hline 33 & $\{100\}$ & $b_{n}=0$ & $a_{\Sigma}=b_{\Sigma}=b_{\Delta}=0$ & $4_{n}, 2_{1}$ & 422 \\
\hline 34 & $\{100\}$ & $b_{n}=0$ & $a_{\Sigma}=b_{\Sigma}=b_{\Delta}=0$ & $4_{n}, \overline{2}_{1}^{\prime}, \overline{2}_{n}^{\prime}$ & $4 / m^{\prime} m^{\prime} m^{\prime}$ \\
\hline 35 & $\{100\}$ & $b_{n}=0$ & $a_{\Sigma}=b_{\Sigma}=b_{\Delta}=0$ & $\overline{4}_{n}^{\prime}$ & $\overline{4}^{\prime}$ \\
\hline 36 & $\{100\}$ & $b_{n}=0$ & $a_{\Sigma}=b_{\Sigma}=b_{\Delta}=0$ & $\overline{4}_{n}^{\prime}, 2_{1}$ & $\overline{4}^{\prime} 2 m^{\prime}$ \\
\hline 42 & $\{111\}$ & $b_{n}=0$ & $a_{\Sigma}=b_{\Sigma}=b_{\Delta}=0$ & $\overline{3}_{n}^{\prime}$ & $\overline{3}^{\prime}$ \\
\hline
\end{tabular}


TABLE (continued

\begin{tabular}{c|c|r|c|c|c}
\hline \hline$k$ & $\{n m l\}$-sample & $\begin{array}{c}\boldsymbol{m}_{1}, \boldsymbol{m}_{2}, \boldsymbol{n}_{\mathrm{W}} \text { and } \boldsymbol{n}_{S}: \\
\text { mutual orientation. }\end{array}$ & $\begin{array}{c}\boldsymbol{m}_{1}, \boldsymbol{m}_{2} \text { and } \boldsymbol{n}_{\mathrm{W}}: \\
\text { mutual orientation }\end{array}$ & $\begin{array}{r}\text { Symmetry } \\
\text { elements }\end{array}$ & $\begin{array}{c}\text { MSC } \\
\text { symbol }\end{array}$ \\
\hline 43 & $\{100\},\{110\}$ & $b_{n}=0$ or $b_{1}=0$ & $a_{\Delta}=b_{\Delta}=a_{\Sigma}=0$ & $\left(1,2_{1}^{\prime}, \overline{2}_{2}, \overline{2}_{n}^{\prime}\right) \times(1, \overline{1})$ & $\mathrm{mm}^{\prime} m^{\prime}$ \\
44 & $\{100\},\{110\}$ & $b_{n}=0$ or $b_{1}=0$ & $a_{\Delta}=b_{\Delta}=a_{\Sigma}=0$ & $1,2_{1}^{\prime}, \overline{2}_{2}, \overline{2}_{n}^{\prime}$ & $\mathrm{mm}^{\prime} 2^{\prime}$ \\
45 & $\{n m l\}^{*}$ & $a_{2}=0$ or $b_{2}=0$ & $a_{\Delta}=b_{\Delta}=a_{\Sigma}=0$ & $1, \overline{1}, 2_{2}, \overline{2}_{2}$ & $2 / m$ \\
46 & $\{n m l\}^{*}$ & $a_{n}=0$ or $b_{n}=0$ & $a_{\Delta}=b_{\Delta}=a_{\Sigma}=0$ & $1, \overline{1}, 2_{n}^{\prime}, \overline{2}_{n}^{\prime}$ & $2^{\prime} / m^{\prime}$ \\
47 & $\{n m l\}^{*}$ & $a_{1}=0$ or $b_{1}=0$ & $a_{\Delta}=b_{\Delta}=0$ & $1, \overline{1}, 2_{1}^{\prime}, \overline{2}_{1}^{\prime}$ & $2^{\prime} / m^{\prime}$ \\
48 & arbitrary & arbitrary & $a_{\Delta}=b_{\Delta}=0$ & $1, \overline{1}$ & $\overline{1}$ \\
49 & $\{n m l\}^{*}$ & $a_{n}=0$ or $b_{n}=0$ & $a_{\Delta}=b_{\Delta}=b_{\Sigma}=0$ & $1, \overline{1}, 2_{n}, \overline{2}_{n}$ & $2 / m$ \\
50 & $\{100\},\{110\}$ & $b_{n}=0$ or $b_{1}=0$ & $a_{\Delta}=b_{\Delta}=b_{\Sigma}=0$ & $1,2_{1}^{\prime}, 2_{2}^{\prime}, 2_{n}$ & $22^{\prime} 2^{\prime}$ \\
51 & $\{100\},\{110\}$ & $b_{n}=0$ or $b_{1}=0$ & $a_{\Delta}=b_{\Delta}=b_{\Sigma}=0$ & $\left(1,2_{1}^{\prime}, 2_{2}^{\prime}, 2_{n}\right) \times(1, \overline{1})$ & $m m^{\prime} m^{\prime}$ \\
53 & $\{111\}$ & $b_{n}=0$ & $a_{\Delta}=b_{\Delta}=b_{\Sigma}=0$ & $32_{n}, 2_{1}^{\prime}$ & $32^{\prime}$ \\
55 & $\{111\}$ & $b_{n}=0$ & $a_{\Delta}=b_{\Delta}=b_{\Sigma}=0$ & $\overline{3}_{n}, \overline{2}_{1}^{\prime}$ & $\overline{3} m^{\prime}$ \\
56 & $\{100\}$ & $b_{n}=0$ & $a_{\Delta}=b_{\Delta}=b_{\Sigma}=0$ & $44_{n}, \overline{2}_{n}$ & $4 / m^{\prime}$ \\
57 & $\{100\}$ & $b_{n}=0$ & $a_{\Delta}=b_{\Delta}=b_{\Sigma}=0$ & $4_{n}, 2_{1}^{\prime}$ & $42^{\prime} 2^{\prime}$ \\
58 & $\{100\}$ & $b_{n}=0$ & $a_{\Delta}=b_{\Delta}=b_{\Sigma}=0$ & $4_{n}, \overline{2}_{1}^{\prime}, \overline{2}_{n}$ & $4 / m m^{\prime} m^{\prime}$ \\
59 & $\{100\}$ & $b_{n}=0$ & $a_{\Delta}=b_{\Delta}=b_{\Sigma}=0$ & $\overline{4}_{n}$ & $\overline{4}$ \\
60 & $\{100\}$ & $b_{n}=0$ & $a_{\Delta}=b_{\Delta}=b_{\Sigma}=0$ & $\overline{4}_{n}, 2_{1}^{\prime}$ & $\overline{4} 2^{\prime} m^{\prime}$ \\
64 & $\{111\}$ & $b_{n}=0$ & $a_{\Delta}=b_{\Delta}=b_{\Sigma}=0$ & $\overline{3}_{n}$ & $\overline{3}$ \\
\hline
\end{tabular}

* $(n m l)$-plates with arbitrary Miller indexes except non-zero values $|n| \neq|m| \neq|l| \neq|n|$.

** At $\left(\boldsymbol{n}_{\mathrm{W}} \boldsymbol{a}_{1}\right)=\left(\boldsymbol{n}_{\mathrm{W}} \boldsymbol{a}_{2}\right)=0$.

*** The possible symmetry elements are rotations around twofold symmetry axes $2_{n}, 2_{n}^{\prime}$ or $2_{1}, 2_{1}^{\prime}$ or else $2_{2}$, $2_{2}^{\prime}$ that are collinear with the unit vectors $\boldsymbol{n}_{\mathrm{W}}$ or $\boldsymbol{a}_{1}$ or else $\boldsymbol{a}_{2}$, respectively, reflections in planes $\overline{2}_{n}, \overline{2}_{n}^{\prime}$ or $\overline{2}_{1}, \overline{2}_{1}^{\prime}$ or else $\overline{2}_{2}, \overline{2}_{2}^{\prime}$ that are normal to the above mentioned vectors, respectively, rotations around three-, four-fold symmetry axes $3_{n}, 4_{n}$ that are collinear with the vector $\boldsymbol{n}_{\mathrm{W}}$, rotations around three-, four-fold inversion symmetry axes $\overline{3}_{n}, \overline{3}_{n}^{\prime}, \overline{4}_{n}, \overline{4}_{n}^{\prime}$ that are collinear with the vector $\boldsymbol{n}_{\mathrm{W}}$, inversion in the symmetry center $\overline{1}, \overline{1}^{\prime}$ and identity 1 . Here an accent at symmetry elements means a simultaneous use of the time reversal operation [6].

General enumeration of MSCs of $0^{\circ}$-DWs contains MSCs with: $k=2,6 \leq k \leq 13,16 \leq k \leq 19, k=22$, $24,26,30,32 ; 43 \leq k \leq 51, k=53 ; 55 \leq k \leq 60$ and $k=64$. The $60^{\circ}-$ and $120^{\circ}$-DWs are represented by MSCs with $k=10,16,18$ and $k=11,13,16$, respectively. The MSCs of the $70.5^{\circ}-, 90^{\circ}$ - (both for $\langle 100\rangle$ and $\langle 110\rangle$ like easy magnetization axis [5]) and $109.5^{\circ}$-DWs are the MSCs with $7<k<13,16<k<18$. The general list of $180^{\circ}$-DWs includes MSCs with $1 \leq k \leq 42$, except for $k=25,28,37-41$.

\section{Conclusions}

The complete collection of $(\mathrm{nml})$-plates with all possible orientations includes the full list of MSCs of $2 \alpha-$ -DWs in cubic $m \overline{3} m$ crystal. For separate $(n m l)$-plates with fixed combination of the Miller indexes this list is limited. Such limitation depends on plate orientation. It is minimal and maximal for the samples with high-symmetry (such as (100)-, (110)- or (111)-plates) and low-symmetry (the $(\mathrm{nml})$-plates, where indexes are non- -zero and have different absolute values) developed surface, respectively. Maximal quantity of MSCs of $2 \alpha$-DWs is for (100)-plates. The MSC with $k=16$ is the MSC of all above-mentioned $2 \alpha$-DWs in arbitrary oriented plate of the cubic $m \overline{3} m$ crystal.

\section{References}

[1] V. Baryakhtar, V. Lvov, D. Yablonsky, J. Exp. Theor. Phys. 87, 1863 (1984).

[2] V. Baryakhtar, E. Krotenko, D. Yablonsky, J. Exp. Theor. Phys. 91, 921 (1986).

[3] R. Vakhitov, A. Yumaguzin, J. Magn. Magn. Mater. 215-216, 52 (2000).

[4] B.M. Tanygin, O.V. Tychko, Physica B, Condens. Matter. 404, 4018 (2009).

[5] A. Hubert, R. Shafer, Magnetic Domains. The Analysis of Magnetic Microstructures, Springer, Berlin 1998.

[6] L. Shuvalov, Modern Crystallography IV: Phys. Prop. Cryst., Springer, Berlin 1988. 